\title{
Anticancer Activity of Oridonin Against Esophageal Cancer Cells Enhanced by Special Electromagnetic Field Treated Water
}

\author{
Jiang $\mathrm{PI}^{1}$, Jin-huan JIANG ${ }^{1}$, Hua JIN ${ }^{1}$, Xin WANG ${ }^{2}$, Yuan $P E N G^{2}$ \\ and Ji-ye CAI ${ }^{1,3, a,{ }^{*}}$
}

${ }^{1}$ State Key Laboratory of Quality Research in Chinese Medicines, Macau University of Science and Technology, Macau, 000853, China

${ }^{2}$ Biological Spectrum Institute, Guangdong Junfeng BFS Technology CO.,LTD

Guangzhou, 510600, China

${ }^{3}$ Department of Chemistry, Jinan University, Guangzhou, 510632, China

atjycai@jnu.edu.cn

${ }^{*}$ Corresponding author

Keywords: Oridonin, Cancer, Special electromagnetic field treated water.

\begin{abstract}
Oridonin, the main pharmacological active substance isolated from Rabdosia Rubescens, has been found to show anti-inflammatory, pro-apoptotic, neurological effects, and excellent anticancer activities. However, the clinical application of oridonin is still limited by its poor solubility. The special electromagnetic field treated water (SEFT water) is served as a kind of high quality drinking water in China. In the present work, the SEFT water was used to test its effects on the anticancer activity of oridonin. And we found that the proliferation inhibition effects of oridonin in EC9706 cells were enhanced in SEFT water dissolved DMEM medium. And the apoptosis induction effects of oridonin in EC9706 cells were also enhanced in SEFT water dissolved DMEM medium, implying that SEFT water could enhance the anticancer effects of oridonin in cancer cells. It was also worth to note that the solubility of oridonin was also enhanced in SEFT water, which might contribute to the enhanced anticancer effects of oridonin in SEFT water dissolved medium for cancer cells.
\end{abstract}

\section{Introduction}

Oridonin, the main pharmacological active substance isolated from Rabdosia Rubescens, has been found to show anti-inflammatory, pro-apoptotic, and neurological effects. And among its biological activities, the excellent anticancer activities of oridonin have been widely reported. It has been reported that oridonin can induce apoptosis or autophagy in various kinds of cancer cells, such as multiple myeloma cells [1], colorectal cancer cells [2], hepatoma carcinoma cell [3], prostate cancer cells [4], cervical carcinoma cells [5] and oesophageal cancer cells [6], indicating oridonin as a very good candidate for cancer treatment.

Water is the most important constitute of human body that participates and plays very critical roles in nearly all biological phenomena. With the increase of life quality, more and more peoples are concerning about the water quality they drink. Based on this, special electromagnetic field treated water (SEFT water) is produced by the treatment of clean water with special electromagnetic fields. In normal conditions, water molecules $\left(\mathrm{H}_{2} \mathrm{O}\right)$ exist as large water molecule clusters, which contribute to the high viscosity, surface tension, melting point and boiling point of water [7]. With 
electromagnetic fields treatment, the large aggregates with more than a dozen of water molecules would be changed to smaller aggregates with fewer water molecules [8]. The obtained SEFT water has been reported to have a lot of advantages beyond than normal water. For example, the conductance of purified water, tap water and sea water are all enhanced after electromagnetic fields treatment [9]. Additionally, SEFT water was also found to enhance the microcirculation of rats, especially to decrease the adhesion of leukocytes $[10,11]$. It was also reported that SEFT water could improve the blood viscosity, in rats, which would also benefit the microcirculation of body [12]. These results demonstrated the better bio- activity of SEFT water than that of normal water, indicating the potential health care effects of SEFT water. However, up to now, whether SEFT water could affect the biological activity of drugs against in vitro cells is still unknown.

In the present work, we investigated the effects of SEFT water on the proliferation inhibition effects and apoptosis induction effects of oridonin on EC9706 cells. The obtained results indicated that SEFT water could both enhance the proliferation inhibition effects and apoptosis induction effects of oridonin in EC9706 cells. And interestingly, we found that SEFT water could significantly increase the solubility of oridonin, which might contribute to the enhanced proliferation inhibition effects and increased apoptosis induction effects of oridonin on cancer cells in SEFT water dissolved DMEM medium.

\section{Materials and Methods}

\section{Materials}

Fetal bovine serum (FBS), penicillin/streptomycin, dulbecco's modified eagle medium (DMEM) powders, and trypsin kit are obtained from Gibco (USA). Oridonin (98\%, HPLC) is obtained from Mingwang biotechnology (China). 3-(4, 5)-dimethylthiazo(-z-y1)-3,5-diphenyt-etrazoliumromide (MTT) and Annexin V-FITC /PI (Annexin V-Fluorescein Isothiocyanate/Propidium Iodide) apoptosis detection kit are purchased from Beyotime Institute of Biotechnology (China). Junfeng BFS water treatment \& healthcare device, which generate special electromagnetic field, is provided by Guangdong Junfeng BFS Technology CO.,LTD (China). Milli-Q water was produced by a Milli-Q purification system (Millipore, German) is used all through the experiments.

\section{Preparation and Characterization of SEFT Water}

SEFT water was produced by treating Milli-Q water (controlled water) using Junfeng BFS water treatment \& healthcare device (Guangdong Junfeng BFS Technology CO.LTD, China)) for six times. The successful preparation of SEFT water was determined by testing the full width ${ }^{17} \mathrm{O}$ peaks for water molecules, and the conductivity for both SEFT water and controlled water. The ${ }^{17} \mathrm{O}$ peaks of SEFT water and controlled water were recorded by ARX-400 nuclear magnetic resonance spectrometer (Bruker, German). The conductivity of SEFT water, controlled water and plasma (Provided by the national biological products research institute) were tested by electrical bridge (Shenghuida, China) and three-electrode conductivity cell (Mettler toledo, Switzerland). The oxygen dissolution of controlled water and SEFT water was determined by dissolved oxygen meter (YSI, USA). And the oxygen dissolution in controlled water was set as $100 \%$ to calculate the differences of oxygen dissolution in controlled and SEFT water. 


\section{Preparation of Milli-Q Water Dissolved DMEM Medium and SEFT Water Dissolved DMEM Medium}

$1.35 \mathrm{~g}$ DMEM powders (Gibco, USA) and $0.37 \mathrm{~g} \mathrm{NaHCO}_{3}$ (Sigma, USA) are dissolved in $100 \mathrm{ml}$ Milli-Q water. Then, HCL is used to adjust the $\mathrm{pH}$ value of the medium to 7.05-7.15. Then, FBS (Gibco, USA) and penicillin/streptomycin (Gibco, USA) are added into the medium to obtain $10 \%$ FBS, $100 \mathrm{U} / \mathrm{mL}$ penicillin and $100 \mathrm{~g} / \mathrm{mL}$ streptomycin Milli-Q water dissolved DMEM medium. For SEFT water dissolved DMEM medium, $1.35 \mathrm{~g}$ DMEM powders and $0.37 \mathrm{~g} \mathrm{NaHCO}_{3}$ are dissolved in $100 \mathrm{ml}$ SEFT water and its $\mathrm{pH}$ value is adjusted to $7.05-7.15$ by HCL (Sigma, USA). Then, FBS and penicillin/streptomycin are added into the medium to obtain 10\% FBS, 100 $\mathrm{U} / \mathrm{mL}$ penicillin and $100 \mathrm{~g} / \mathrm{mL}$ streptomycin SEFT water dissolved medium. Therefore, SEFT water treatment is obtained by treating cells with Milli-Q water dissolved DMEM medium (control) and SEFT water dissolved DMEM medium (SEFT water treated) to treat the cells with or without oridonin, respectively.

\section{Cell Culture}

Human oesophageal cancer EC9706 cell line is purchased from tumor cell library of Chinese Academy of Medical Sciences (BeiJing, China). Cells are cultured with Milli-Q water dissolved DMEM medium supplemented with $10 \%$ FBS, $100 \mathrm{U} / \mathrm{mL}$ penicillin, and $100 \mathrm{~g} / \mathrm{mL}$ streptomycin in a humidified atmosphere of $5 \% \mathrm{CO}_{2}$ at $37{ }^{\circ} \mathrm{C}$.

\section{Cell Viability Measurement}

MTT (Beyotime, China) assay was used to test the cell viability of EC9706 cells exposed to oridonin in different kinds of mediums. The cells were seeded into 96 well plates with a density of $5 \times 10^{3}$ cells/well for $24 \mathrm{~h}$ incubation. After that, different concentrations of oridonin dissolved in Milli-Q water dissolved DMEM medium and SEFT water dissolved DMEM medium were added into the plate wells for $24 \mathrm{~h}$ incubation, respectively. After oridonin treatment, MTT reagent $(10 \mathrm{uL}, 5 \mathrm{mg} / \mathrm{mL})$ was then added into each well for $4 \mathrm{~h}$ incubation, the medium was removed, and the cells were suspended in $150 \mathrm{uL}$ DMSO to incubate for $10 \mathrm{~min}$. A spectrophotometer (TECAN, Switzer-land) was used to test absorption at $570 \mathrm{~nm}$.

\section{Cancer Cell Apoptosis Detection}

Annexin V-FITC/PI apoptosis detection kit (Beyotime, China) was used to detect the apoptosis of oridonin treated EC9706 cells in different kinds of mediums according to the manufacturer's instructions. The cells were seeded into 6 well plates with a density of $1 \times 10^{5}$ cells/well for $24 \mathrm{~h}$. After that, different concentrations of oridonin dissolved in Milli-Q water dissolved DMEM medium and SEFT water dissolved DMEM medium were added into the plate wells for $24 \mathrm{~h}$ incubation, respectively. After incubated with oridonin, EC9706 cells were harvested, washed triple with PBS, suspended in Annexin $\mathrm{V}$ binding buffer, and incubated with FITC-labeled Annexin V and PI for $5 \mathrm{~min}$ at room temperature in dark. Then, the samples were immediately analyzed by Flow cytometry (BD, USA).

\section{Solubility Measurement}

The solubility of oridonin was detected by the absorption of saturated oridonin solution both in Milli-Q and SEFT water using UV-Vis spectroscopy. The saturated solution of oridonin was obtained by adding oridonin into Milli-Q water and SEFT water to make sure that some insoluble oridonin could be observed in the water. Then, the UV 
absorption of the supernants from the saturated oridonin solution was determined at a wavelength of $242 \mathrm{~nm}$. The differences of solubility were expressed as the ratio between the absorption value of saturated oridonin solution in SEFT water and the absorption value of saturated oridonin solution in Milli-Q water. And the solubility of oridonin in Milli-Q water was set as $100 \%$ to calculate the differences of solubility for oridonin in Milli-Q and SEFT water.

\section{Results and Discussion}

\section{Decreased Width of Nuclear Magnetic Resonance Absorption Peak for SEFT Water}

As shown in Fig. $1,{ }^{17} \mathrm{O}$ nuclear magnetic resonance analysis could be used to study the relative size of water molecules formed aggregates [7]. Using ${ }^{17} \mathrm{O}$ nuclear magnetic resonance, we also analyzed the SEFT water and controlled water (without special electromagnetic field treatment). The full width at half maximum intensity of ${ }^{17} \mathrm{O}$ for controlled water was $112.7 \mathrm{~Hz}$. After the water was treated with special electromagnetic fields, its full width at half maximum intensity of ${ }^{17} \mathrm{O}$ was 62.6 $\mathrm{Hz}$. The much higher ${ }^{17} \mathrm{O}$ full width at half maximum intensity of controlled water demonstrated that the cluster of controlled water molecules were much larger than the cluster of SEFT water molecules.

A

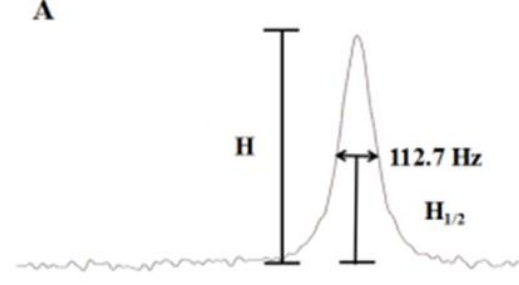

B

Fig.1 Nuclear magnetic resonance analysis of (A) control water and (B) SEFT water.

\section{Enhanced Conductivity and Dissolved Oxygen of SEFT Water}

The conductivity of controlled water, SEFT water and plasma was also determined and the results were shown in Fig.2, which demonstrated that the conductivity of SEFT water was higher than that of controlled water. Additionally, we also found that the dissolved oxygen of controlled water increased from $100 \%$ to $105.3 \pm 2.3 \%$ for SEFT water (Fig.3). These results suggested that the conductivity and dissolved oxygen ability of SEFT water were higher than that of controlled water.

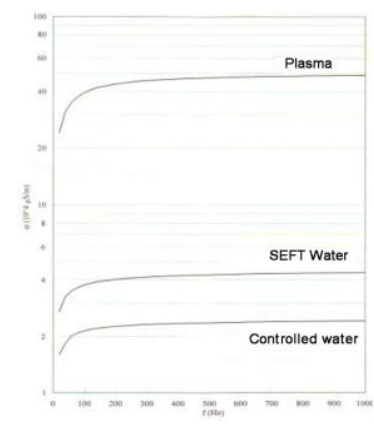

Fig.2 Conductivity of control water and SEFT water. 


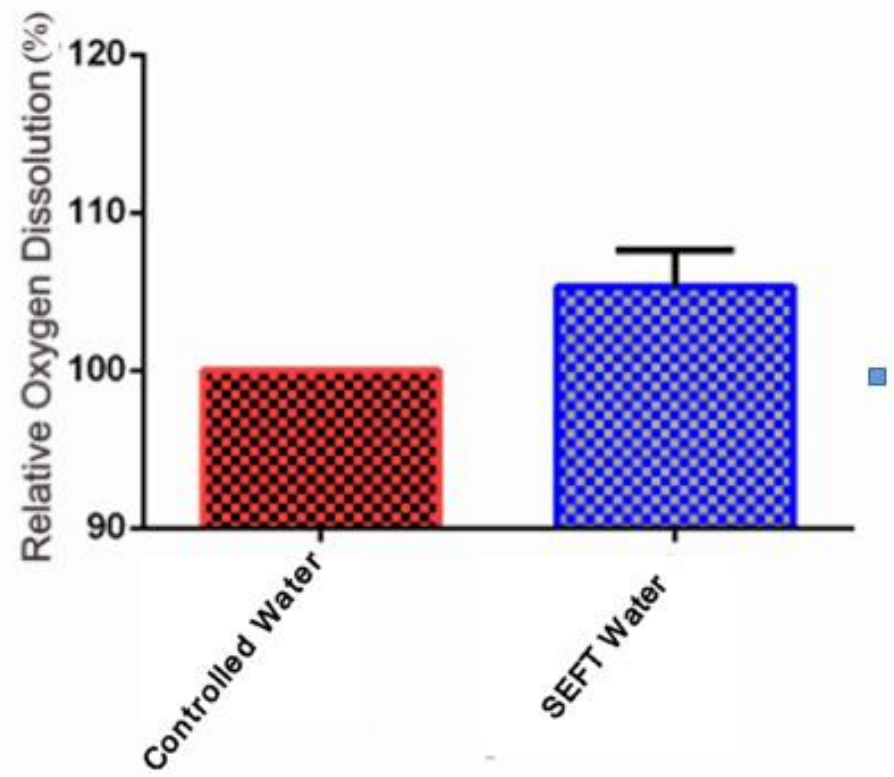

Fig.3 Dissolved oxygen in control water and SEFT water, $n=3$.

\section{SEFT Water Enhance the Proliferation Inhibition Effects of Oridonin in EC9706 Cells}

The effects of SEFT water on the proliferation inhibition effects of oridonin against EC9706 cells were determined by MTT assay. As shown in Fig.4, the viability of cells decreased from $100 \%$ for controlled cells to $97.6 \pm 1.1 \%, 88.5 \pm 1.2 \%, 76.5 \pm 3.9 \%$, $64.2 \pm 5.9 \%$ and $39.5 \pm 9.2 \%$ after $10 \mu \mathrm{M}, 20 \mu \mathrm{M}, 30 \mu \mathrm{M}, 40 \mu \mathrm{M}$ and $50 \mu \mathrm{M}$ oridonin treatment in Milli-Q water dissolved DMEM medium, respectively. However, the viability of cells decreased from $100 \%$ for controlled cells to $93.5 \pm 2.9 \%, 76.8 \pm 6.4 \%$, $61.6 \pm 7.4 \%, 48.1 \pm 9.1 \%$ and $18.9 \pm 2.8 \%$ after $10 \mu \mathrm{M}, 20 \mu \mathrm{M}, 30 \mu \mathrm{M}, 40 \mu \mathrm{M}$ and $50 \mu \mathrm{M}$ oridonin treatment in SEFT water dissolved DMEM medium, respectively. These results demonstrated that oridonin in SEFT water dissolved DMEM medium caused stronger inhibition effects on EC9706 cell proliferation, which suggested that SEFT water could enhance the proliferation inhibition effects of oridonin in EC9706 cells.

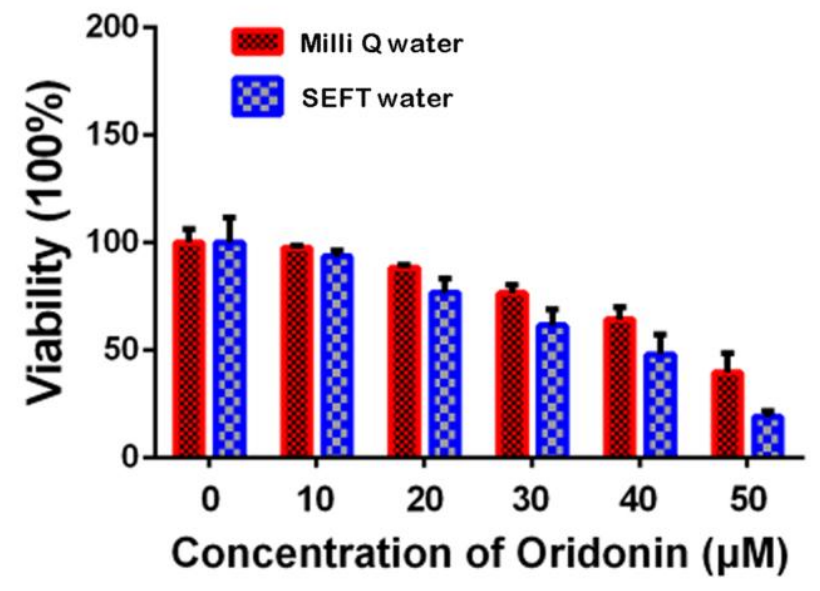

Fig.4 Cell viability of ECY/U6 cells treated with ditterent concentratıons of orıdonin in Milli-Q water dissolved DMEM medium and SEFT water dissolved DMEM medium, n=3. 


\section{SEFT Water Enhance the Apoptosis Induction Effects of Oridonin in EC9706 Cells}

Using flow cytometry, the effects of SEFT water on the apoptosis induction effects of oridonin in EC9706 cells were further determined by Annexin V-FITC and PI assay. As shown in Fig.5A-E, $30 \mu \mathrm{M}$ oridonin treated EC9706 cells showed no significant differences in the apoptotic cells between Milli-Q water dissolved DMEM medium and SEFT water dissolved DMEM medium. However, the apoptotic cells (including early and late apoptosis) in $50 \mu \mathrm{M}$ oridonin treated EC9706 cells increased from $66.38 \%$ for Milli-Q water dissolved DMEM medium to $76.37 \%$ for SEFT water dissolved DMEM medium. By statistical analysis (Fig. $5 \mathrm{H}$ ), we also found that the apoptotic cells increased from $6.4 \pm 1.3 \%$ for controlled cells to $12.2 \pm 4.8 \%$ and $58.1 \pm 8.4 \%$ after $30 \mu \mathrm{M}$ and $50 \mu \mathrm{M}$ oridonin treatment in Milli-Q water dissolved DMEM medium, respectively. And the apoptotic cells increased from $6.7 \pm 1.3 \%$ for controlled cells to $15.4 \pm 6.2 \%$ and $69 \pm 6.6 \%$ after $30 \mu \mathrm{M}$ and $50 \mu \mathrm{M}$ oridonin treatment in SEFT water dissolved DMEM medium, respectively. These results demonstrated that SEFT water enhanced the apoptosis induction effects of oridonin in EC9706 cells, especially in high concentration oridonin treated cells.
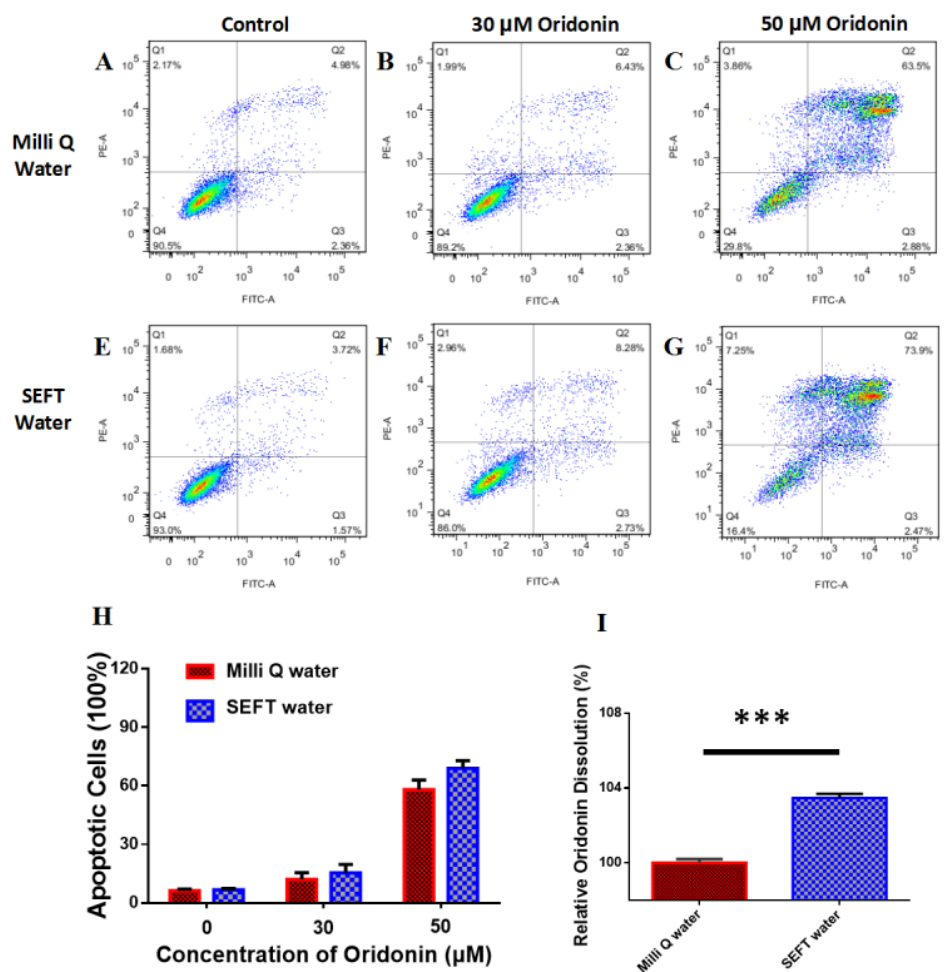

Fig.5 (A-E) Apoptosis of EC9706 cells treated with different concentrations of oridonin in Milli-Q water dissolved DMEM medium and SEFT water dissolved DMEM medium. (H) Statistical analysis of the apoptosis of EC9706 cells treated with different concentrations of oridonin in Milli-Q water dissolved

DMEM medium and SEFT water dissolved DMEM medium, $n=3$. (I) Differences of solubility for oridonin in Milli-Q water and SEFT water, $\mathrm{n}=3, * * * \mathrm{p}<0.005$.

\section{SEFT Water Enhance the Solubility of Oridonin}

To further determine the potential mechanism of how SEFT water enhance the proliferation inhibition effects and apoptosis induction effects of oridonin in EC9706 
cells, we also determined the effects of SEFT water on the solubility of oridonin. As shown in Fig.5I, the solubility of oridonin increased from 100\% for Milli-Q water to $103.5 \pm 0.2 \%$ for SEFT water. The obtained results demonstrated a nearly $3.5 \%$ increase of solubility for oridonin, which implied that the enhancement of anticancer effects of oridonin in EC9706 cells might due to the increased solubility of oridonin in SEFT water dissolved DMEM medium.

\section{Conclusions}

In the present work, we found that SEFT water had much narrower half maximum intensity of ${ }^{17} \mathrm{O}$ nuclear magnetic resonance, higher conductivity and higher dissolved oxygen than that of Milli-Q water. And additionally, we also demonstrated that the proliferation inhibition effects of oridonin on EC9706 cells in SEFT water dissolved DMEM medium were stronger than that of Milli-Q water dissolved DMEM medium. And SEFT water dissolved DMEM medium could also increase oridonin induced apoptotic EC9706 cells, also implying that SEFT water could enhance the anticancer effects of oridonin in cancer cells. It was also worth to note that the solubility of oridonin was also enhanced in SEFT water, which might contribute to the enhanced anticancer effects of oridonin in SEFT water dissolved medium for cancer cells. These results collectively suggested that SEFT water could be served as a kind of potential health care products that might enhance the anticancer activity of hard -to-dissolve anticancer drugs.

\section{Acknowledgement}

This work is supported by the project of Jinan University (No. 40115085) and the Macao Science and Technology Development Fund (No 028/2014/A1).

\section{Abbreviations}

SEFT

DMEM

$\mathrm{UV}-\mathrm{V}$ is

FITC

PI

DMSO
Special electromagnetic field treated water

Dulbecco's modified eagle media

Ultraviolet visible

Fluorescein isothiocyanate

Propidium Iodide

Dimethy lsulfoxide

\section{References}

[1] R Zeng, Y Chen, S Zhao, GH Cui . Acta Pharmacol Sin, 2012; 33: 91-100.

[2] FH Gao, F Liu, W Wei, LB Liu, MH Xu, ZY Guo, W Li , B Jiang, YL Wu . Int J Mol Med, 2012; 29: 649-55.

[3] J Huang ,LJ Wu , Tashiro S, Onodera S, Ikejima T. J Pharmacol Sci, 2008; 107 : 370-9.

[4] LH Ye, WJ Li , XQ Jiang, YL Chen, SX Tao, WL Qian ,JS He . Anat Rec, 2012; 295: 417-422.

[5] YH Zhang, YL Wu, Tashiro S, Onodera S, Ikejima T. Acta Pharmacol Sin, 2011;

32: 1266-75. 
[6] JH Chen, SB Wang, DY Chen, GY Chang, QF Xin, SJ Yuan, ZY Shen . Chin J Clin Oncol, 2007; 4: 16-20.

[7] FZ Li , X Zhang, MJ Lv . Acta Scien Circum, 2004; 24: 6-9.

[8] SX Liu . Change of Property of Magnetized Water and its Effect on Protein molecular and Escherichia-coli Protein. Tianjing University, 2007.

[9] LV Kr, BH Di . Pro Mod Biomed, 2007; 7: 361-77.

[10] YY Liu , XM Zhao , FY Liu . Ch J Microcircu, 2002; 12: 17-8.

[11] YY Liu , XM Zhao ,FY Liu . . Ch J Clin Rehabil, 2004; 8: 479-81.

[12] YY Liu, Y Han, SR Zheng . Ch J Microcircu, 2007;13: 23-4. 